\title{
Klippel-Trenaunay Syndrome: a case report with brief review of literature
}

\author{
Mohammad Iqbal Zea, Mohammad Hanif, Mohammad Habib, Ahmed Ansari \\ Department of Surgery, Jawaharlal Nehru Medical College, Aligarh, U P, 202002, India
}

Corresponding author:

Mohammad Iqbal Zea

Department of Surgery, Jawaharlal Nehru Medical College

Aligarh Muslim University, Aligarh, India

E-mail: iqbal945@gmail.com

\section{Key words:}

Brodie-Trendelenburg test, KlippelTrenaunay Syndrome, Tendelenberg test, Varicose veins

\begin{abstract}
Background: Klippel-Tranuanay Syndrome is a rare sporadic disease characterized by clinical triad of capillary malformation; soft tissue and bony hypertrophy; and atypical varicosity. Although this syndrome was first described more than hundred years ago, exact incidence has not been estimated yet. Several theories have been postulated to describe its pathogenesis. Clinical presentation of this syndrome is protean ranging from minimal asymptomatic disease to life threatening bleeding and embolism. Management of this syndrome includes careful diagnosis, prevention and treatment of complications.
\end{abstract}

Main observation: We are presenting a case of Klippel-Tranuanay Syndrome in a 19-year-old male patient presenting with varicosity in lateral aspect of right leg. On careful examination other components of the syndrome were found. Patient was kept on yearly follow up.

Conclusion: Usually patients with Klippel-Tranuanay Syndrome present with complication. This case shows that there is also a need for medical solutions in patients who seek advise for cosmetic reasons.

\section{Introduction}

Complexity of vascular malformation made it difficult not only to classify ${ }^{1}$ it but also to diagnose its rare subtypes. Additionally their sporadic occurrence increases the chances of mistakes due to wrong diagnosis. Klippel-Trenaunay syndrome (KTS) belongs to such type of disease appears with a wide range of capillary, venous, soft tissues and bony abnormalities. Diagnosis of this embryological malformation is purely clinical, which plays a pivotal role in its management. Treatment of KTS is conservative unless complications occur. ${ }^{1}$ We are presenting a case of uncomplicated KTS with brief review of world literatures.

\section{Case report}

A 19-year-old male patient presented to the surgery department of Jawaharlal Nehru Medical College, Aligarh in the month of April, 2009 with complaints of dilated veins over outer aspect of right lower limb since birth. He reported that the dilatation over the limb increased during walking and standing. It subsided when lying down with the limb raised. Cutaneous pigmentation was present over the right knee. There was no history of ulceration, difficulty in walking, pain in legs, paresthesia, or cellulitis.

On examination multiple varicose veins were present over the lateral aspect of right lower limb extending from above the ankle to the upper thigh (Fig. 1).

Brodie-Trendelenburg test showed sapheno-femoral vaIve to be competent. Pratt's test demonstrated competent deep venous system. Trendelenberg test showed competent perforators. Cutaneous hyperpigmentation with raised margins (port wine stains) was present over the lateral aspect of right lower limb, expressed more around the knee (Fig. 2). On measurement right limb was $75 \mathrm{~cm}$ from greater trochanter to medial malleolus while left limb was $73 \mathrm{~cm}$. Right lower limb was found to be $2 \mathrm{~cm}$ longer than left lower limb.

Color Doppler of the patient revealed multiple varicosities all over the leg \& lower thigh region, few showing thrombus formation within competent deep venous system. Mid thigh \& medial leg perforators could be visualized \& found normal in size \& flow. Sapheno-femoral \& sapheno-popliteal junctions were patent (Fig. 3). 
On the basis of above triad of port wine stain, limb hypertrophy and lateral varicosity diagnosis of Klippel-Trenaunay syndrome was made. The patient was treated conservatively by elastic compression stockings. He was advised to keep the limb elevated where possible. He is being followed up yearly.

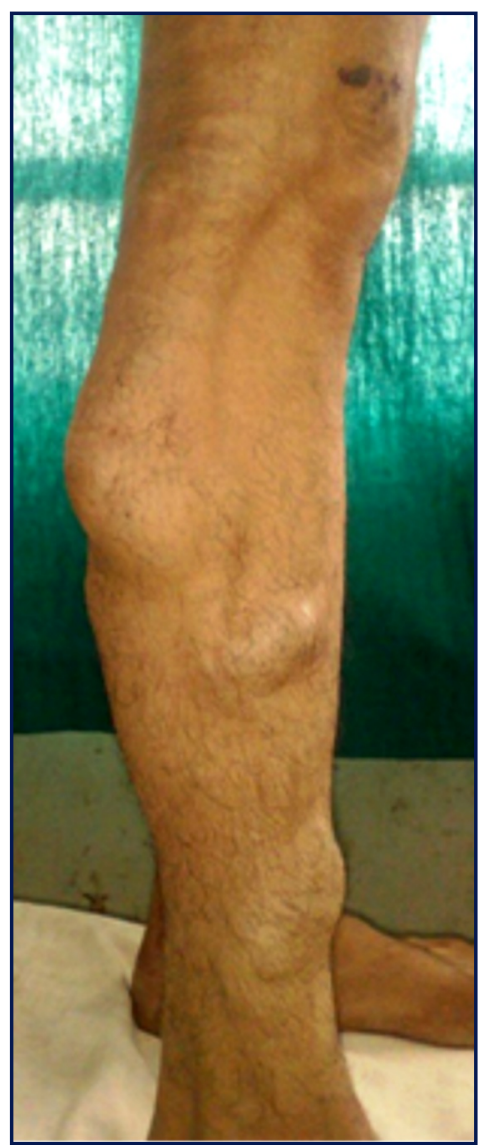

Figure 1

Dilated lateral marginal vein.

\section{Brief review of literature}

Historic overview: About a century ago, two French physicians Maurice Klippel and Paul Trenaunay described two patients with haemangiomatous lesions of the skin associated with asymmetric soft tissue and bone hypertrophy, and coined the term 'naevus variqueux osteohypertrophique'. ${ }^{2}$ This rare syndrome characterized by clinical triad of (1) capillary malformation (port-wine stain); (2) soft tissue and bony hypertrophy; and (3) atypical mostly lateral varicosity, was later termed as Klippel Tranuanay Syndrome (KTS). KTS should be distinguished from Parkes-Weber syndrome, a mixed, high-flow, high shunt arteriovenous malformation ${ }^{3}$, since clinical features, management and prognosis of these two entities are distinctly different. KTS has a wide spectrum of presentation, from truncular to extratruncular, from infiltrating to limited forms, containing primarily three anomalous vascular elements: veins, capillaries and lymphatics.

Etiology: The aetiology of KTS is unknown. KTS is most commonly a sporadic event. Several theories have been proposed, including (1) Servelle's theory of a primary obstruction of the venous system resulting in venous hypertension and therefore development of abnormal venous pathways and tissue overgrowth; (2) failure of regression of the lateral limb bud vein; and (3) alteration of the tight balance between angiogenesis and vasculogenesis, which is controlled by numerous genes, among other theories. ${ }^{4}$ Berry et al in 1998 speculated that in KTS there is an alteration in vascular remodelling, perhaps at the level of altered angiopoietin- 2 antagonism. ${ }^{5}$ Various case reports of KTS are present in world literatures. Still, incidence and genetic predisposition of this rare disease has not been established.

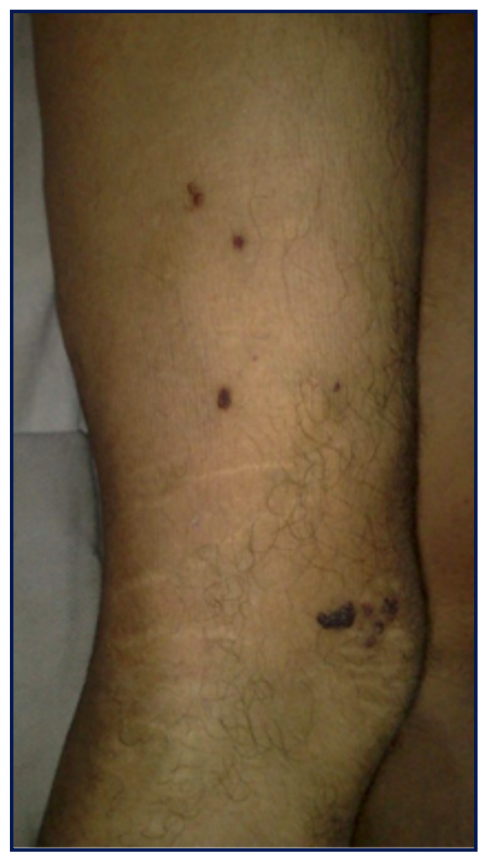

Figure 2

Port wine stains around knee.

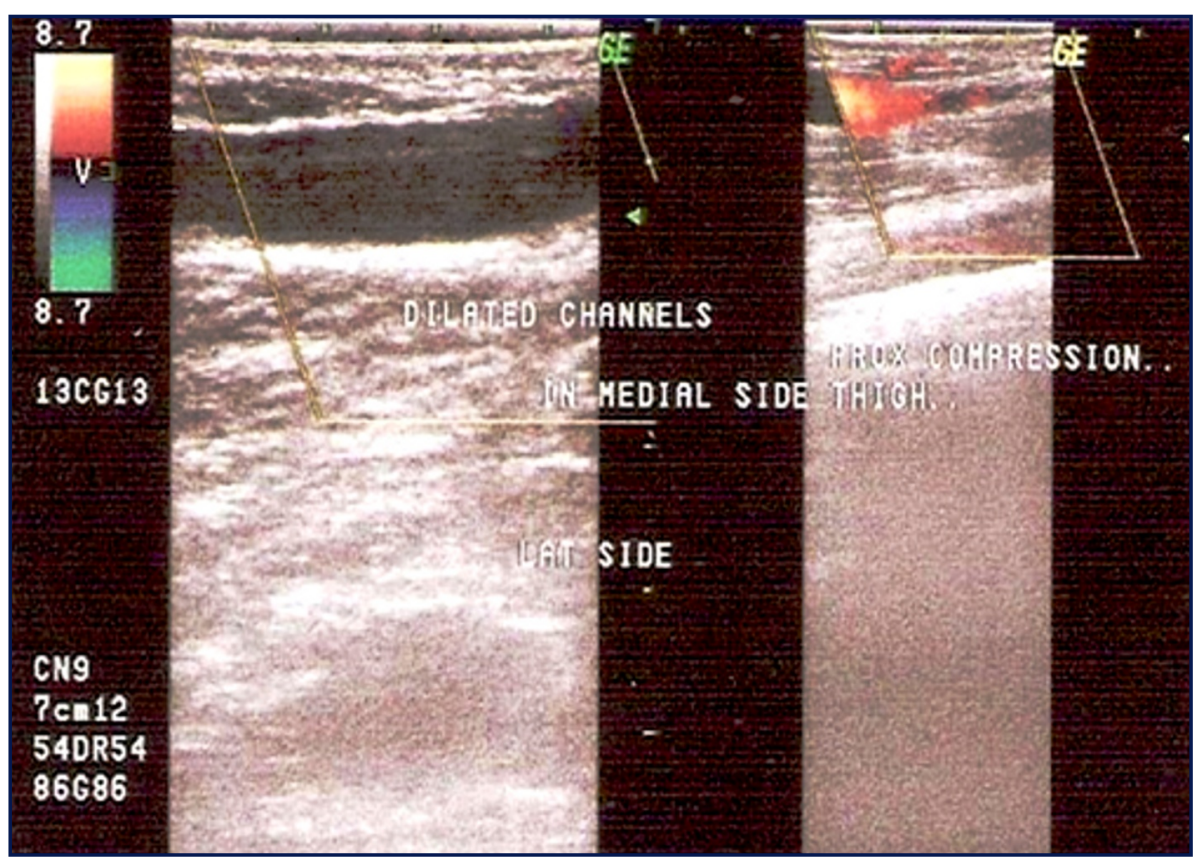

Figure 3

Color Doppler showing hugely dilated superficial valve less vein. 
Clinical presentation: The classic clinical triad includes capillary malformations (port wine stain), a usually longer and larger extremity because of soft tissue and bone hypertrophy, and atypical mostly lateral superficial varicosity. Patients with at least two of the three cardinal features have been classified as having an incomplete form of KT syndrome. Deep vein anomalies like venous hypoplasia to frank aneurysm, valve hypoplasia to avalvulia have been decribed. Lymphatic malformations have also been prevalent.

The venous malformations frequently present as persistence of embryonic veins, of which the lateral marginal vein (the vein of Servelle) has been the most typical findingfound in $68-80 \%$ of patients. ${ }^{6}$ This vein originates from the lateral aspect of the foot and courses upwards along the lateral border of the leg. The vein is usually thick walled and strong, it is located immediately under the skin and it is incompetent along its entire length due to the absence of venous valves. Drainage is either into a lateral branch of the profunda femoris vein or into the internal iliac vein. ${ }^{7}$ Another embryonic vein sciatic vein has also been described in KTS. 8 This may provide the main drainage for lower limb. Varicose veins and venous malformations can involve abdominal and pelvic organs. Genitourinary manifestations may present as intrapelvic and retroperitoneal vascular malformations and affect the penis, scrotum, vagina or vulva, and bladder. Bleeding from capillary or venous malformations or persistent embryonic veins may occur through defects in the skin or mucosa, or the patient can have intramuscular or retroperitoneal haematoma, haematuria, rectal bleeding, intracerebral or intraspinal haemorrhage. Gl tract involvement may be more common in KTS than previously believed because most of cases remain unrecognized without overt symptoms. ${ }^{9}$ Large cutaneous lesions may sequester platelets leading to Kasabach-Merritt syndrome, a type of consumptive coagulopathy. Rarely, patients with KTS can have intraosseous vascular malformations.

The bony abnormalities may affect all bones in an extremity or limited to one or two bones. Single limb involvement is found in $80-85 \%$ patients. Apart from hypertrophy or sometimes hypotrophy of bones other deformities includes macrodactyly, syndactyly, split hand deformity, phalangeal agenesis and dislocation of hip joint. ${ }^{10}$ Soft tissue hypertrophy may be limited to a localized mass on the back or chest, or it can be diffuse involving an entire arm or leg. Lymphatic deformities found in upto $70 \%$ of cases include primary lymphaedema to cystic hygroma or have lymphangiectasia associated with reflux of chyle. ${ }^{7}$

Clinical presentation of patients with KTS has a wide spectrum from incomplete, mild forms of port wine stains and few varicose veins causing only cosmetic deformity to severe disability associated with massive limb overgrowths, chronic pain syndrome, skin infections, arthritis ${ }^{11}$ thromboembolism and life-threatening pelvic or recurrent rectal bleeding from venous malformations. ${ }^{12}$

Diagnosis: Diagnosis of KTS is mainly clinical. Investigations in KTS should focus on evaluation of the type, extent and severity of the malformation, and on confirming the absence of any clinically significant arteriovenous shunting.

Physical examination of limb and pelvic lesions is complemented by detailed colour duplex scanning of the venous system of the leg to establish patency, incompetence, thrombosis, arteriovenous shunting and any anomalies such as hypoplasia.

Plain X-rays of the long bones (scanograms) are most helpful to measure bone length. MRI is helpful in differentiating bone, fat, muscle hypertrophy and lymphedema.

Contrast venography and computed tomography scans are required to evaluate deep venous system and collateral, when ablation of dilated superficial embryonic vein has been planned. ${ }^{13}$

Management: A multidisciplinary approach to treatment and prevention of possible complications of KTS including paediatrician, internist, phlebologist, paediatric, orthopaedic, plastic and vascular surgeons, an interventional radiologist, cardiologist or vascular internist and a physical therapy physician provides optimal care for the patient.

Prevention of venous thrombo-embolism with anticoagulation or inferior vena cava filter is as important as prevention of repeated episodes of cellulitis and lymphangitis in those with associated lymphoedema or as treatment of the symptomatic vascular anomalies.

The absolute indications of treatment are haemorrhage, infections, acute thromboembolism or refractory ulcers. The management of KTS has been largely conservative. Compression therapy has been the mainstay of conservative treatment in the form of an elastic garment or compression bandage. This has been beneficial in managing both lymphoedema and chronic venous insufficiency. Local wound care, compression dressings, special orthopaedic footwear and lifestyle modification may also be required to manage activities of daily living and improve the function of the limb. ${ }^{14}$

A number of minimal invasive procedures are adopted for management of venous abnormalities. It includes pulse-dye laser therapy for port wine stain. ${ }^{5}$ Serial sclerotherapy with absolute alchohol has been used with good success but can cause nerve injury and cutaneous damage. ${ }^{15}$ Foam sclerotherapy with sodium tetra decyl sulphate and polidocanol has been a low risk and effective treatment of superficial venous disease. ${ }^{16,17}$ Patient's cosmetic satisfaction with multiple session foam sclerotherapy has been reported upto $71.5 \% .{ }^{18}$

Cellulitis and thrombophlebitis can be managed with analgesics, elevation, antibiotics, and corticosteroids. In patients with a history of recurrent cellulitis, intermittent or prophylactic antibiotics may be considered. Anticoagulant therapy is indicated in acute thrombosis and prophylactically prior to surgical procedures. ${ }^{19}$

Surgery is reserved selectively for symptomatic patients with KTS those are not candidate for less invasive treatment. Vascular interventions must be preceded by careful evaluation of the extent of malformations and patency of the deep system. Difficult Intubation due to facial asymmetry 
and central nervous system hemangiomas may cause intraoperative bleeding further increasing complication rates. ${ }^{20}$ In cases with normal deep veins, complete surgical resection of the marginal vein is the best form of treatment. This vein may have very large perforators to the deep veins, so adequate exposure of the veins is recommended, especially for large perforators to be ligated. If deep veins are hypoplastic marginal vein can be resected because deep veins are able to dilate spontaneously to almost normal size after resection. ${ }^{21}$ But aplastic deep vein is absolute contraindication for resection of marginal vein. The use of subfascial endoscopic perforator surgery (SEPS) in patients with large incompetent perforating veins and venous ulcers has been useful and some patients benefit from deep venous reconstructions. ${ }^{22}$

Regarding limb hypertrophy, heel inserts are generally sufficient for limb discrepancies of $1.5 \mathrm{~cm}$ or less. For greater discrepancies, orthopedic surgery may be considered. For overgrowth of one limb epiphyseodesis and for severe arthritis total knee arthroplasty has shown good results. ${ }^{11}$ Amputation of affected limb may be required when the large size of the limb had interfered with the activities of daily. 23

Gastrointestinal and urogenital involvement is not as much less that was previously thought. Now it has been found that gastrointestinal involvement is up to $20 \%$ and urological involvement is around $30 \%$. These patients usually require to endoscopic cauterization, but sometimes refractory bleeding may require colonic resections. 24,25

\section{Conclusion}

The manifestations of KTS are protean and may be confused with Parkes-Weber syndrome. The absence of clinically significant arteriovenous shunting distinguishes KTS from Parkes-Weber syndrome. ${ }^{26}$ Exact incidence and pathogenesis is still unknown. Diagnosis is purely clinical. Management is conservative with life long follow up. Appropriate multidisciplinary approach of management is required as disease affects multiple organs.

\section{References}

1. Young AE. Combined vascular malformations. In Mulliken JB, Young AE, eds. Vascular Birthmarks: Hemangiomas and malformations. Philadelphia: Saunders, 1988; 246-274.

2. Klippel M, Trenaunay P. Du naevus variquex osteohypertrophique. Arch Gen Med. 1900; 3: 641-672.

3. Weber FP. Angioma-formation in connection with hypertrophy of limbs and hemi-hypertrophy. Br J Dermatol. 1907; 19: 231.

4. Tian X, Liu M, Kadaba R, et al. Positional cloning of a novel angiogenic factor gene: VG5Q mutations cause susceptibility to KTS. Nature. 2004; 427: 640-645.

5. Berry SA, Peterson C, Mize W, et al. Klippel-Trenaunay syndrome. Am J Med Gen. 1998; 79: 319-326.
6. Kim YW, Lee BB, Cho JH, et al. Haemodynamic and clinical assessment of lateral marginal vein excision in patients with a predominantly venous malformation of the lower extremity. Eur J Vasc Endovasc Surg. 2007; 33: 122-127.

7. Servelle M. Klippel-Trenaunay syndrome: 768 operated cases. Ann Surg. 1985; 201: 365-373.

8. Cherry KJ, Gloviczki P, Stanson AW. Persistent sciatic vein: diagnosis and treatment of a rare condition. J Vasc Surg. 1996; 23: 490-497.

9. Cha SH, Romeo MA, Neutze JA. Visceral manifestations of Klippel-Trenaunay syndrome. Radiographics. 2005; 25: 1694-1697.

10. McGrory BJ, Amadio PC. Klippel-Trenaunay syndrome: orthopaedic considerations. Orthop Rev. 1993; 22: 41-50.

11. Catre M G, Kolin A, Waddell J P. Total knee arthroplasty in Klippel-Trenaunay syndrome. Can J Surg. 2005; 48(6): 494495.

12. Jacob AG, Driscoll DJ, Shaughnessy WJ, Stanson AW, Clay RP, Gloviczki P. Klippel-Trenaunay syndrome: spectrum and management. Mayo Clin Proc. 1998; 73: 28-36.

13. Gloviczki P, Driscoll DJ. Klippel-Trenaunay syndrome: current management. Phlebology. 2007; 22: 291-298.

14. Lee BB, Do YS, Byun HS, Choo IW, Kim DI, Huh SH. Advanced management of venous malformation with ethanol sclerotherapy: mid-term results. J Vasc Surg. 2003; 37: 533-538.

15. Burrows PE, Mason KP. Percutaneous treatment of low flow vascular malformations. J Vasc Interv Radiol. 2004; 15: 431 445.

16. Coleridge-Smith P. Chronic venous disease treated by ultrasound guided foam sclerotherapy. Eur J Vasc Endovasc Surg. 2006; 32: 577-583.

17. Cabrere J, Cabrera Jr J, Olmeda AG, Redondo P, Treatment of Venous Malformations With Sclerosant in Microfoam Form. Arch Dermatol. 2003; 139: 1409-1416.

18. Nitecki S, Bass A. Foam Sclerotherapy in Klippel-Trenaunay Syndrome. IMAJ. 2007; 9: 72-75.

19. Lee A, Driscoll D, Gloviczki P, Clay R, et al. Evaluation and Management of Pain in Patients with Klippel-Trenaunay Syndrome: A Review. Pediatrics. 2005; 115(3): 744-749.

20. Marin RMP, Collada JCG, Martinez AIG, Serrano EMM, Aguilar JMM. Anaesthetic management of Klippel Trenuany Syndrome and attendant gastrointestinal heamorrhage. Minerva Aneasthiol. 2007; 73: 187-190.

21. Mattassi R, Vaghi M. Management of the marginal vein: current issues. Phlebology. 2007; 22: 283-286.

22. Noel AA, Gloviczki P, Cherry Jr KJ, Rooke TW, Stanson AW, Driscoll DJ. Surgical treatment of venous malformations in Klippel-Trenaunay syndrome. J Vasc Surg. 2000; 32: 840-847.

23. Abdul-Rahman NR, Mohammad KF, Ibrahim S. Gigantism of the lower limb in Klippel-Trenaunay syndrome: anatomy of the lateral marginal vein. Singapore Med J. 2009; 50(6): e223-e225.

24. Husmann DA, Rathburn SR, Driscoll DJ. Klippel-Trenaunay syndrome: incidence and treatment of genitourinary sequelae. J Urol. 2007; 177(4): 1244-9.

25. Wilson $\mathrm{CL}$, Song $\mathrm{LM}$, Chua $\mathrm{H}$, et al. Bleeding from cavernous angiomatosis of the rectum in Klippel-Trénaunay syndrome: report of three cases and literature review. Am J Gastroenterol. 2001; 96: 2783-2788.

26. Biesecker LG, Happle R, Mulliken JB, et al. Proteus syndrome: diagnostic criteria, differential diagnosis, and patient evaluation. Am J Med Genet. 1999; 84: 389-395. 